\title{
On the Appearance of Thyroid-like Structures in Ovarian Cysts.
}

By R. HaMruton Bell, M.A., M.B., B.C. (Cantab.), F.R.C.S., M.R.C.P. Obstetric Tutor and Registrar to St. Thomas's Hospital, etc.

Two cases of this rare condition have occurred within the last few years at St. Thomas's Hospital. I am indebted to Dr. Walter Tate for permission to bring them before the Society.

The cases had no particular interest from a clinical standpoint. They were regarded before operation as examples of ordinary ovarian cysts, and it was only microscopic examination which led to the discovery of the peculiar character of the growths.

E.B., aged 32, was in hospital October, 1902. She was a married woman, and had five children. Menstruation had been irregular since January, 1902, and nine weeks before admission she had severe pains, and passed clots, possibly a mole. A month later there was severe hæmorrhage, which recurred a week before admission. Concurrently with the menstrual disturbance an abdominal swelling was noticed, which at times was tender. On examination the swelling was seen to be centrally situated, rounded, freely mobile, and fluctuating. It reached to the level of the umbilicus. Per vaginam it could be felt in front of the uterus and depressing the anterior vaginal wall.

The uterus was first curetted, and then the abdomen opened and a cyst of the left ovary removed. A small cyst in the right ovary was punctured, and the ovary left in situ.

The cyst removed was handed to me for examination. It was multilocular. The largest loculus had been opened by trocar and cannula during the operation. It contained $1 \frac{1}{2}$ pints of dark reddishbrown fluid, and some brownish blood clot. The specific gravity of the fluid was $1 \cdot 035$, and it became solid on boiling. The cyst wall was thin and smooth, with some blood-stained patches, but with no intra-mural hæmorrhage. The rest of the tumour consisted of a number of small cysts containing fluid of varied consistence, and also a small mass of firm tissue, about the size of a walnut, which I thought would prove to be more or less solid adenomatous growth. It was sent to the clinical laboratory for microscopical examination, and the report returned was "Thyroid tissue. ? Secondary thyroidal carcinoma."

* Read at a meeting of the Obstetrical Society of London, June, 1905. 


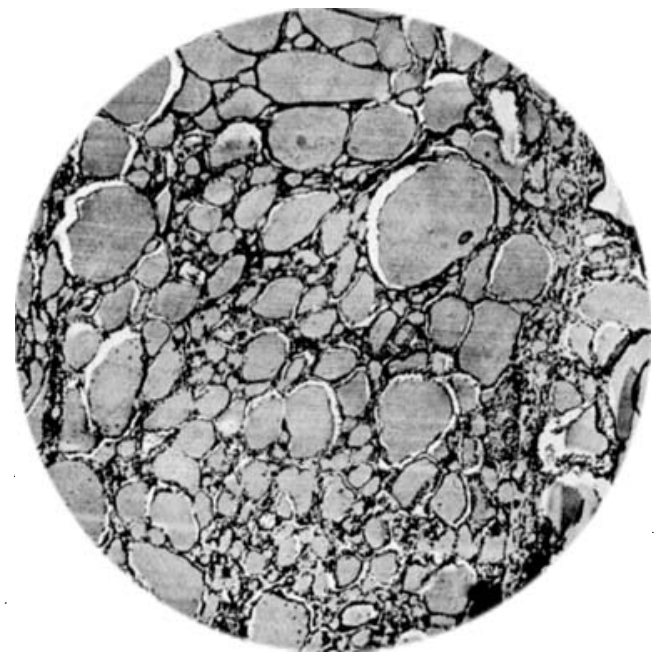

Section under low power, from case occurring in 1902.

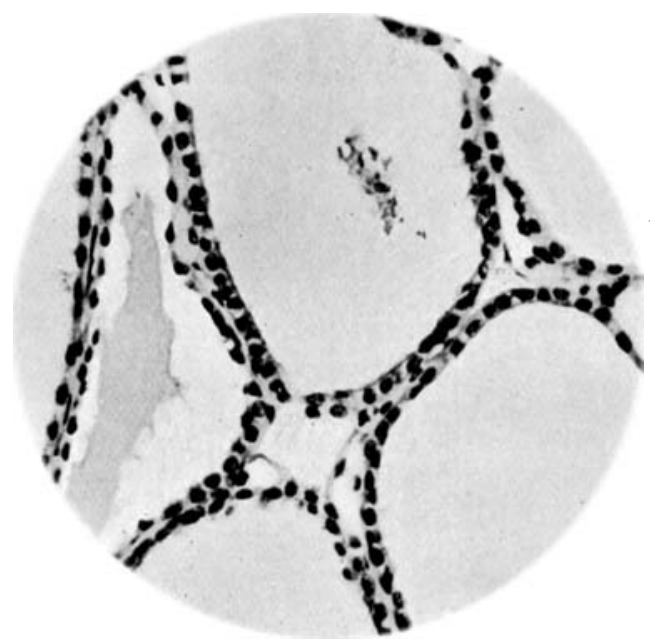

Small portion under high power to show flat cubical epithelium, from case occurring in 1904. 
A careful examination of the patient's neck was made, but nothing abnormal discovered. Convalescence was uninterrupted, and the patient was allowed to leave the hospital, having been warned to return at once should any abnormal symptoms occur, especially in the neck or abdomen.

The second case occurred two years later, November, 1904.

M.L., single, aged 52. Menstruation regular till five years before admission, when the menopause occurred. She complained of no symptoms beyond increase in the size of the abdomen and constipation. The tumour was larger than in the other case, reaching well above the umbilicus. Ovariotomy was performed, and a loose twist of the pedicle was noted. It was a multilocular ovarian cyst of the right side, and the largest loculus contained eight pints of dark chocolate-coloured fluid. As in the other case, there was some staining of the cyst wall, but no intra-mural hæmorrhage. I noted numerous deposits of calcerous particles in the cyst wall, the largest being the size of an incisor tooth. At the base of the cyst near the pedicle was a congeries of small cysts, and another more solid mass, very similar to that noted in the first case, but containing a few calcerous particles. The report from the clinical laboratory was "Tumour suggests a metastatic thyroid carcinoma."

In this case the neck was distinctly enlarged, especially on the right side. The enlargement was soft and had no definite outline, but suggested a goitre. The patient stated that her mother had a similar enlargement. Above this soft enlargement there was on the right side, at the level of the thyroid cartilage, a small harder lump, which moved on deglutition. It appeared quite separate from other structures in the neck. It had not the exact consistence of an enlarged gland and the possibility of its being an accessory thyroid suggested itself.

The question of an exploratory operation upon the neck was discussed, and it was clear that it was of importance to obtain the subsequent history of the previous case. I succeeded with some difficulty in tracing the patient to Peterborough, and learnt in a letter from her husband that her general health had steadily improved during the last two years, that she had at times had a certain amount of pain in the abdomen, but that the occasions were less frequent now than formerly, and that she had had no trouble whatever in her neck. The second patient was then allowed to leave the hospital, but was to be kept under observation. 
Microscopical appearances. These may be shortly put as being those of the thyroid gland. In the first section may be seen a large number of circular or rounded spaces, very varied in size, and for the most part completely filled with colloid material. The spaces are lined with a flat cubical epithelium. In a few of the smaller spaces the flat epithelium is seen without the colloid material. At places there is a certain amount of connective tissue, with some colloid material in the lymph spaces, but for the most part the connective tissue is sparse.

On the outer surface of the specimen a distinct columnar epithelium can be seen at places, and especially one small patch, almost separate from the main mass of the section, which is lined throughout by columnar epithelium, but contains in its interior several small spaces holding colloid material, and lined by flattened cubical epithelium.

In the more recent section the appearances are very similar to those already described, but the colloid material is more shrunken, and does not fill the spaces. These are, however, lined with the typical flat epithelium. The slight difference is probably due to different methods of preparation. There is much more connective tissue in this section, usually of a loose character. As before, some colloid may be seen in the lymph spaces.

The number of cases in which this thyroid appearance has been observed in the ovary is comparatively small. Probably not more than half-a-dozen have been recorded. It is difficult to come to a conclusion as to the proper explanation of it. I have already mentioned in recording the cases that on both occasions the report sent up to the ward from the clinical laboratory suggested the possibility of the growth being secondary to a primary carcinoma of the thyroid gland. The subsequent history of the first case, already referred to, renders this suggestion most improbable. But while it seems clear that the growth is not a metastasis from a thyroid carcinoma, it may possibly be secondary to a simple goitre, or even arise from a normal thyroid gland. Cases have been recorded in the Transactions of the Pathological Society, in which extensive secondary growth have arisen in the calvaria, having their origin not in a carcinoma, but in a simple parenchymatous enlargement of the thyroid. I refer to the cases of Morris ${ }^{1}$ and Coats. ${ }^{2}$ Moreover, Oderfeld and Steinhaus ${ }^{3}$ have stated that "normal human thyroid may grow into blood-vessels, and thus give rise to osseous metastases of apparently thyroid tissue." 
So far as I am aware, there is no recorded case of a secondary growth in soft parts arising from a simple goitre, but if we are to accept the view that normal thyroid tissue may enter the blood stream, there seems to be no reason why it should not reach the ovary. This is the explanation of the phenomenon put forward by Karl Kretschmar ' in his latest paper. Previously he had expressed the opinion that these tumours were endotheliomata, a view that has been adopted by others. It has also been suggested that they were growths of a teratomatous nature.

Neither of these two latter explanations appeals to me; nor, indeed, am I convinced that the appearances, though so similar to those of the thyroid gland, are really due to anything more than a peculiar colloid degeneration of an ordinary cystic adenoma of the ovary. The flat cubical epithelium may be produced by the pressure of the secreted colloid material. Edmunds ${ }^{5}$ has shown that in Graves's disease the colloid in the vesicles has partly or wholly disappeared, and that the secreting cells, instead of being cubical, are columnar, and that these changes are practically identical with those of compensatory hypertrophy, as seen in animals after experimental partial removal. This seems to indicate that the flat thyroid epithelium becomes columnar as soon as relieved from the pressure of the colloid material. It is, therefore, not very fantastic to suppose that where an extensive colloid degeneration occurs in the ovary, the epithelium may become flattened merely from pressure. Through the kindness of Dr. Powell White, my attention was drawn to a section of a kidney, which was very similar to those $I$ have described in this paper. It was taken from a shrivelled and disorganised organ, the change in the kidney being no doubt due to complete obliteration of the ureter. The thyroid was normal, and it is hard to believe that the thyroid appearances in this disorganised kidney were due to any metastasis of the thyroid gland, or to any active tumour formation, or, indeed, to anything else than a colloid degeneration. This kidney section, therefore, tends to confirm the view that the thyroid-like structures in the ovary are really due to a degenerative change. I will, however, conclude with a summary of the various possible explanations of this rare occurrence.

1. That it is a metastatic growth from a primary thyroidal carcinoma.

2. That it is a secondary growth from a normal thyroid gland, or from a simple goître.

3. That it is a growth of teratomatous nature. 
4. That it is an endothelioma.

5. That it is a peculiar colloid degeneration of an ordinary cystic adenoma of the ovary.

As indicated above, my own inclination is to accept, tentatively at least, the last of these suggestions, but I bring forward the specimens in the hope that some Fellows of wider pathological and clinical experience may throw fresh light upon them, and perhaps definitely determine their pathological position.

\section{ADDENDUM.}

The microscopic sections were submitted to the Pathology Committee of the Obstetrical Society. Their report was read at the meeting of the Society on Wednesday, July 5th. It was in the following terms:-

"We have examined these microscopic sections, and agree with Dr. Bell's report thereon. We consider that the tissue in question so closely resembles the structure of adult thyroid gland that we are unable to distinguish between them. The sections show no other structures, suggesting a teratoma.

Sections from three other multilocular ovarian tumours were shown by Mr. Targett, and corresponded exactly in microscopical structure with those exhibited by Dr. Bell. These sections also showed no evidence of a teratomatous structure.

Sections from an undoubted ovarian teratoma were shown by Dr. Herbert Williamson. These exhibited a tissue which, in its histological characters, exactly resembles the thyroid-like structure of the preceding specimens.

The evidence before us is not sufficient to justify us in expressing an opinion as to the origin of this thyroid-like tissue."

This report was signed by Dr. Griffith (Chairman), Mr. Corrie Keep (Secretary), Mr. Bellingham Smith, Mr. Targett, Dr. Herbert Williamson, and myself.

\section{RFF mRENCES.}

1. Path. Soc. Trans., 1880, xxxi.,

2. Path. Soc. Trans., 1887, sxxviii.

3. Central. für Path., 1901. Bd. xii., 209.

4. Monats. für Geburts. und Gynäkol. Bd. xix., 389 and 546.

5. Lancet, May 11, 18 and 25, 1901. 